\title{
Correction to: VLSI-Based Pipeline Architecture for Reversible Image Watermarking by Difference Expansion with High-Level Synthesis Approach
}

\author{
Subhajit Das ${ }^{1} \cdot$ Sudip Ghosh $^{3}$ - Nachiketa Das ${ }^{4}$. Santi P. Maity ${ }^{5}$. \\ Hafizur Rahaman ${ }^{5}$ - Reshmi Maity ${ }^{2}$ - Niladri Maity ${ }^{2}$ \\ Published online: 12 November 2018 \\ ○) Springer Science+Business Media, LLC, part of Springer Nature 2018
}

\section{Correction to: Circuits, Systems, and Signal Processing (2018) 37:1575-1593 https://doi.org/10.1007/s00034-017-0609-3}

The original version of the article unfortunately contained an error in the author group. Four authors were not included in the original version.

Dr. Sudip Ghosh, Dr. Nachiketa Das, Dr. Santi P. Maity and Dr. Hafizur Rahaman are newly included as second, third, fourth and fifth authors in the author group. Dr. Reshmi Maity and Dr. Niladri Maity are now listed as sixth and seventh authors in the author group.

The original article can be found online at https://doi.org/10.1007/s00034-017-0609-3.

Niladri Maity

mzut133@mzu.edu.in

Subhajit Das

subhajitdas151@gmail.com

Reshmi Maity

mzut138@mzu.edu.in

1 Department of Electronics and Communication Engineering, National Institute of Technology,

Aizawl 796 012, India

2 Department of Electronics and Communication Engineering, Mizoram University, Aizawl 796 004, India

3 School of VLSI Technology, Indian Institute of Engineering Science \& Technology (IIEST), Shibpur, Howrah, West Bengal 711 103, India

4 Indian Maritime University, Kolkata Campus, Kolkata 700 088, India

5 Department of Information Technology, Indian Institute of Engineering Science \& Technology (IIEST), Shibpur, Howrah, West Bengal 711 103, India 